Article

\title{
Clinical Characteristics and Treatment Outcomes of Pathologically Confirmed Aspergillus Nodules
}

\author{
Noeul Kang ${ }^{1,+}{ }^{\infty}$, Jiyeon Park ${ }^{2,+}$ and Byung Woo Jhun ${ }^{1, *}$ \\ 1 Division of Pulmonary and Critical Care Medicine, Department of Medicine, Samsung Medical Center, \\ Sungkyunkwan University School of Medicine, Seoul 06351, Korea; varsagod0314@gmail.com \\ 2 Department of Medicine, Samsung Medical Center, Sungkyunkwan University School of Medicine, \\ Seoul 06351, Korea; science0528@gmail.com \\ * Correspondence: byungwoo.jhun@gmail.com; Tel.: +82-2-3410-3429; Fax: +82-2-3410-3849 \\ + These authors contributed equally to this work.
}

Received: 19 June 2020; Accepted: 8 July 2020; Published: 10 July 2020

\begin{abstract}
Aspergillus nodules represent a subtype of chronic pulmonary aspergillosis, but details on their characteristics and outcomes are limited. We evaluated 80 patients with pathologically confirmed Aspergillus nodules between January 2009 and December 2016. The median age of the patients was 59 years, and $46(58 \%)$ were women. Seventy-three (91\%) patients were surgically diagnosed with Aspergillus nodules and the remaining seven (9\%) patients were diagnosed by percutaneous transthoracic needle biopsy. The median long-axis diameter of nodules was $22 \mathrm{~mm}$, and nodules had an internal cavity in $49(61 \%)$ patients. Spiculation and calcification were observed in $20 \%$ and $39 \%$ of patients, respectively. Ninety percent (18/20) of nodules showed uptake on positron emission tomography. Serum Aspergillus precipitin IgG antibody was positive in 42\% (10/24) of tested patients. Seventy-three (91\%) patients underwent surgery without $(n=58)$ or with $(n=15)$ adjuvant antifungal therapy, and the remaining seven $(9 \%)$ patients received antifungal therapy alone $(n=5)$ or no treatment $(n=2)$. Three patients experienced postoperative pulmonary complications: pneumothorax, hemoptysis, and acute lung injury ( $n=1$ each). There was no recurrence during the median follow-up period of 36.8 months. In conclusion, surgery could be a treatment strategy worth considering for most Aspergillus nodules. However, given that our study population was heterogeneous, further well-designed studies are need.
\end{abstract}

Keywords: chronic pulmonary aspergillosis; nodule; Aspergillus nodule; treatment

\section{Introduction}

Chronic pulmonary aspergillosis (CPA) is a slowly progressing pulmonary infection caused by Aspergillus species, typically Aspergillus fumigatus [1,2]. In general, CPA occurs in middle-aged and elderly immunocompetent individuals with chronic pulmonary diseases (e.g., mycobacterial infection, obstructive lung disease, sarcoidosis, or previous history of thoracic surgery) and there is some in-vitro evidence that patients with CPA may have subtle immune defects that confer predisposition to disease [3-5]. CPA shows poor prognosis and, as it is associated with multiple respiratory comorbidities, such as tuberculosis, this became a substantial burden in the developing world [6,7]. CPA typically comprises cavity formation with para-cavitary infiltrates but may appear in various other forms. In recent European guidelines, CPA was divided into several phenotypes: simple aspergilloma/nodules, chronic cavitary or fibrosing pulmonary aspergillosis, and a subacute invasive form [1].

Aspergillus nodules represent an uncommon subtype of CPA with single or multiple nodular lesions, with or without cavitation, most of which are smaller than $3 \mathrm{~cm}$ [1]. However, the clinical and radiological manifestations of Aspergillus nodules are nonspecific, and this form of Aspergillus 
infection is challenging to differentiate from other pulmonary diseases in nodular form, especially lung cancer [8,9]. Indeed, most Aspergillus nodules are mistaken for malignancy and are diagnosed based on histological findings after surgical resection [10-12]. Moreover, there is limited evidence to support the use of serum Aspergillus precipitin IgG antibody test for the diagnosis of Aspergillus nodules, although it is recommended as a keystone for the diagnosis of CPA [1,13-15]. In addition, there is a lack of data regarding the prognosis of Aspergillus nodules.

Therefore, it is important to understand the detailed features of Aspergillus nodules in clinical practice, especially to avoid unnecessary interventions. However, previous publications have been mostly limited to case reports; only minimal data are available regarding the outcomes of Aspergillus nodules under the current definition. Hence, the present study was performed to determine the clinical characteristics and treatment outcomes of pathologically confirmed Aspergillus nodules.

\section{Materials and Methods}

\subsection{Study Population}

We retrospectively screened consecutive adult patients (older than 20 years of age) with Aspergillus nodules, which were pathologically confirmed by surgical resection or percutaneous transthoracic needle biopsy (PCNB) between January 2009 and December 2016 at Samsung Medical Center (a 1979-bed referral hospital in Seoul, Republic of Korea). Aspergillus nodules were defined as discrete, small, round, focal opacities on chest computed tomography $(\mathrm{CT})$, which were further divided into two groups according to the absence or presence of internal cavitation (i.e., non-cavitary nodules and cavitary nodules, respectively) [16]. Patients with other subtypes of CPA (e.g., simple aspergilloma, chronic cavitary or fibrosing pulmonary aspergillosis, and subacute invasive disease) were excluded. Finally, 80 patients with Aspergillus nodules were included in the analysis. After surgical resection or PCNB was done, patients were followed-up with either chest X-rays or CT scans at least once in the out-patient clinic. The antifungal agents were used at the discretion of the attending physician. During the follow-ups, a relapse was defined as increased in size or the recurrence of the nodule. The Institutional Review Board of Samsung Medical Center approved the review and publication of information obtained from the patients' records (approval no. 2019-09-036-001). The requirement for informed consent was waived because of the retrospective nature of the study.

\subsection{Clinical and Laboratory Evaluation}

Clinical and demographic characteristics of the patients (e.g., age, sex, smoking habit, body mass index, and comorbidities) were collected. Data regarding inflammatory markers at the time of diagnosis of Aspergillus nodules (e.g., white blood cell WBC] count, erythrocyte sedimentation rate [ESR], and C-reactive protein [CRP]) were analyzed. Fungal culture results of sputum, bronchoalveolar lavage fluid, or tissue at diagnosis were also evaluated. Tests for serum Aspergillus precipitin IgG antibody and/or serum Aspergillus galactomannan antigen were performed at the discretion of the attending physician at the time of diagnosis. The presence of serum Aspergillus precipitin IgG antibody was evaluated using an A. fumigatus IgG ELISA kit (IBL International, Hamburg, Germany). The results were reported as positive $(>12 \mathrm{U} / \mathrm{mL})$, negative $(<8 \mathrm{U} / \mathrm{mL})$, or equivocal $(8-12 \mathrm{U} / \mathrm{mL})$. Serum Aspergillus galactomannan antigen was assessed using a Platelia Aspergillus antigen kit (Bio-Rad, Hercules, CA, USA) and index values were reported as positive $(>0.55)$, negative $(<0.45)$, or equivocal $(0.45-0.55)$ [17].

\subsection{Radiological and Histological Evaluation}

All patients underwent chest CT within one month before the diagnosis of Aspergillus nodules. Two of the authors (N.K. and J.P.) reviewed the CT results. The maximal long-axis diameter of the nodule was measured in the axial view in the lung setting. The following CT findings were analyzed: location, margin of nodules, distribution, the presence of calcification and satellite lesions. A central lesion was defined as a lesion which was located inside the half of the longest diameter of thorax in the 
coronal view of chest CT scan. Likewise, peripheral lesion was defined when it was located outside of the half of the longest diameter. All disagreements were resolved by discussion and subsequent consensus. Results of ${ }^{18} \mathrm{~F}$-fluorodeoxyglucose positron emission tomography (FDG-PET) for Aspergillus nodules were available for 20 patients, and a maximum standardized uptake value $\left(\mathrm{SUV}_{\max }\right)>2.5$ was considered significant. Expert lung pathologists reviewed all pathology specimens. Special staining with methenamine silver stain and Periodic Acid-Schiff stain was performed in addition to routine H\&E staining. Special stains were used to identify specific microorganisms.

\subsection{Statistical Analysis}

Data are presented as medians (interquartile ranges) for continuous variables and numbers (percentages) for categorical variables. For the comparison of characteristics between patients with non-cavitary nodules and patients with cavitary nodules, Student's $t$-test was used for continuous variables and the Pearson Chi-squared test was used for categorical variables. All tests were two-sided, and a $p<0.05$ was considered statistically significant. All analyses were performed using Stata software (version 14.0; Stata Corporation, College Station, TX, USA).

\section{Results}

\subsection{Baseline Characteristics}

The characteristics of the 80 patients with pathologically confirmed Aspergillus nodules are presented in Table 1 . Seventy-three patients (91\%s) were surgically diagnosed with Aspergillus nodules, and the remaining seven patients $(9 \%)$ were confirmed by PCNB without surgery. Thirty-one patients $(39 \%)$ had non-cavitary nodules, while 49 patients $(61 \%)$ had cavitary nodules. The median age of the study population was 59 years (interquartile range, $48-65$ years), and $46(58 \%)$ were women. More than $70 \%$ of patients had never smoked. The most common underlying disease was previous pulmonary tuberculosis $(49 \%)$, followed by bronchiectasis $(14 \%)$, diabetes mellitus $(13 \%)$, and malignancy $(11 \%)$. No patients were treated with steroids, including inhaled corticosteroids. Nearly three-quarters $(77 \%)$ of the patients had respiratory symptoms, most commonly, hemoptysis $(n=54,68 \%)$. Notably, $23 \%$ of the patients had no symptoms at diagnosis.

The median WBC count and ESR were $12,400 / \mu \mathrm{L}$ and $23 \mathrm{~mm} / \mathrm{h}$, respectively. Of patients for whom serum Aspergillus galactomannan antigen $(n=26)$ and serum Aspergillus precipitin IgG antibody $(n=24)$ data were available, positive rates were $31 \%(8 / 26)$ and $42 \%(10 / 24)$, respectively. Fungus culture was positive in $26 \%$ of 46 patients who underwent fungus culture using respiratory specimens, with positive results in 50\% (6/12) of sputum, 17\% (2/12) of bronchoalveolar lavage fluid, and 50\% (6/12) of lung tissue specimens. Overall, there were no significant differences in clinical or laboratory findings between patients with non-cavitary nodules and patients with cavitary nodules, except for WBC counts $(8700 / \mu \mathrm{L}$ vs. $12,900 / \mu \mathrm{L}$, respectively, $p=0.029)$.

\subsection{Radiological and Histological Characteristics of Aspergillus Nodules}

The radiological characteristics of Aspergillus nodules are summarized in Table 2, and the typical findings of Aspergillus nodules with or without an internal cavity are shown in Figures 1 and 2, respectively. Most (98\%) of the patients had a solitary nodule, whereas $2 \%$ had multiple nodules. The median long-axis diameter of all nodules on chest CT was $22 \mathrm{~mm}$ (interquartile range, 16-31 mm). The nodules were located in the right, left, upper, and lower lobes at similar rates. Approximately half of the nodules (51\%) were distributed in peripheral areas. Spiculation (or lobulation) and calcification were observed in $20 \%$ and $39 \%$ of the nodules, respectively. Only $48 \%$ of all nodules were visible on chest radiographs. Of the 20 nodules for which FDG-PET/CT data were available, 90\% (18/20) had relatively high uptake $\left(\mathrm{SUV}_{\max }>2.5\right)$. However, there were no significant differences in radiological characteristics between patients with non-cavitary nodules and patients with cavitary nodules. 
Table 1. Characteristics of patients with pathologically confirmed Aspergillus nodules.

\begin{tabular}{|c|c|c|c|c|}
\hline Characteristics & $\begin{array}{l}\text { Total } \\
(n=80)\end{array}$ & $\begin{array}{l}\text { Non-Cavitary Nodules } \\
(n=31)\end{array}$ & $\begin{array}{l}\text { Cavitary Nodules } \\
(n=49)\end{array}$ & $p$-Value \\
\hline Age, years & $59(48-65)$ & $61(50-70)$ & $56(48-62)$ & 0.058 \\
\hline Female & $46(58)$ & $15(48)$ & $31(63)$ & 0.190 \\
\hline Never smoker & $57(71)$ & $23(74)$ & $34(69)$ & 0.644 \\
\hline Body mass index, $\mathrm{kg} / \mathrm{m}^{2}$ & $24.0(22.1-25.6)$ & $24.0(22.4-25.9)$ & $23.9(22.1-25.6)$ & 0.280 \\
\hline Previous pulmonary tuberculosis & $39(49)$ & $12(39)$ & $27(55)$ & 0.153 \\
\hline Bronchiectasis & $11(14)$ & $6(19)$ & $5(10)$ & 0.322 \\
\hline Nontuberculous mycobacteria & $6(8)$ & $2(6)$ & $4(8)$ & 1.000 \\
\hline COPD/asthma & $3(4)$ & $2(6)$ & $1(2)$ & 0.556 \\
\hline Interstitial lung disease & $3(4)$ & $1(3)$ & $2(4)$ & 1.000 \\
\hline Diabetes mellitus & $10(13)$ & $5(16)$ & $5(10)$ & 0.498 \\
\hline Chronic liver disease & $4(5)$ & $1(3)$ & $3(6)$ & 1.000 \\
\hline Chronic kidney disease & $2(3)$ & 0 & $2(4)$ & 0.519 \\
\hline Malignancy $\mathbb{T I}$ & $9(11)$ & $6(19)$ & $3(6)$ & 0.082 \\
\hline \multicolumn{5}{|l|}{ Symptoms at diagnosis $^{+}$} \\
\hline Hemoptysis & $54(68)$ & $17(55)$ & $37(76)$ & 0.054 \\
\hline Cough & $32(40)$ & $9(29)$ & $23(47)$ & 0.111 \\
\hline Sputum & $5(6)$ & $2(6)$ & $3(6)$ & 1.000 \\
\hline No symptoms & $18(23)$ & $10(32)$ & $8(16)$ & 0.096 \\
\hline \multicolumn{5}{|l|}{ Laboratory findings } \\
\hline $\mathrm{WBC} / \mu \mathrm{L}$ & $\begin{array}{l}12,400 \\
(7600-14,800)\end{array}$ & $\begin{array}{l}8700 \\
(6100-14,100)\end{array}$ & $\begin{array}{l}12,900 \\
(9900-15,000)\end{array}$ & 0.029 \\
\hline $\mathrm{ESR}, \mathrm{mm} / \mathrm{h}(n=42)$ & $23(14-42)$ & $25(15-38)$ & $20(11-44)$ & 0.936 \\
\hline $\mathrm{CRP}, \mathrm{mg} / \mathrm{dl}(n=63)$ & $0.11(0.04-0.41)$ & $0.12(0.03-0.41)$ & $0.11(0.05-0.26)$ & 0.054 \\
\hline Positive serum galactomannan $(n=26)$ & $8 / 26(31)$ & $4 / 8(50)$ & $4 / 18(22)$ & 0.491 \\
\hline Positive serum Aspergillus antibody $(n=24)$ & $10 / 24(42)$ & $2 / 8(25)$ & $8 / 16(50)$ & 0.301 \\
\hline Fungus culture $(n=46)^{\S}$ & $12(26)$ & $2(4)$ & $10(22)$ & 0.115 \\
\hline Sputum & $6 / 12(50)$ & $1 / 2(50)$ & $5 / 10(50)$ & 0.397 \\
\hline Bronchial washing & $2 / 12(17)$ & 0 & $2 / 10(20)$ & 0.519 \\
\hline Lung tissue & $6 / 12(50)$ & $1 / 2(50)$ & $5 / 10(50)$ & 0.397 \\
\hline
\end{tabular}

Data are presented as number (\%) or median (interquartile range). ${ }^{\mathbb{I I}}$ Colorectal cancer $(n=4)$, stomach cancer $(n=2)$, breast cancer $(n=1)$, cholangiocarcinoma $(n=1)$, and lung cancer $(n=1) .{ }^{+} 30$ patients had more than one symptom at diagnosis. $\S$ One patient had positive culture results from both sputum and bronchoalveolar lavage fluid specimens. Another patient had positive culture results from both sputum and lung tissue specimens. COPD, chronic obstructive pulmonary disease; CRP, c-reactive protein; ESR, erythrocyte sedimentation rate; WBC, white blood cells.

Table 2. Radiological and histological findings of Aspergillus nodules.

\begin{tabular}{|c|c|c|c|c|}
\hline Findings & $\begin{array}{l}\text { Total } \\
(n=80)\end{array}$ & $\begin{array}{l}\text { Non-Cavitary Nodules } \\
(n=31)\end{array}$ & $\begin{array}{l}\text { Cavitary Nodules } \\
(n=49)\end{array}$ & $p$-Value \\
\hline Number of nodules & & & & 1.000 \\
\hline 1 & $78(98)$ & $30(97)$ & $48(98)$ & \\
\hline$\geq 2$ & $2(3)$ & $1(3)$ & $1(2)$ & \\
\hline Size $(\mathrm{mm})^{\mathbb{I}}$ & $22(16-31)$ & $20(15-30)$ & $23(17-34)$ & 0.631 \\
\hline$>3 \mathrm{~cm}$ & $21(26)$ & $6(19)$ & $15(31)$ & 0.198 \\
\hline$\leq 3 \mathrm{~cm}$ & $59(74)$ & $25(81)$ & $34(69)$ & \\
\hline Location & & & & 0.496 \\
\hline Right upper lobe & $20(25)$ & $6(19)$ & $14(29)$ & \\
\hline Right lower lobe & $23(29)$ & $11(35)$ & $12(24)$ & \\
\hline Left upper lobe & $17(21)$ & $5(16)$ & $12(24)$ & \\
\hline Left lower lobe & $20(25)$ & $9(29)$ & $11(22)$ & \\
\hline Distribution of nodules & & & & 0.332 \\
\hline Central & $39(49)$ & $13(42)$ & $26(53)$ & \\
\hline Peripheral & $41(51)$ & $18(58)$ & $23(47)$ & \\
\hline Margin of nodules & & & & 0.491 \\
\hline Smooth & $64(80)$ & $26(84)$ & $38(78)$ & \\
\hline Spiculated or lobulated & $16(20)$ & $5(16)$ & $11(22)$ & \\
\hline Calcification in nodules & $31(39)$ & $9(29)$ & $22(45)$ & 0.156 \\
\hline Satellite lesions & $21(26)$ & $8(26)$ & $13(27)$ & 0.943 \\
\hline Lymph node enlargement $(\geq 1 \mathrm{~cm})$ & $7(9)$ & $3(10)$ & $4(8)$ & 0.815 \\
\hline Visible on chest radiographs & $38(48)$ & $14(45)$ & $24(49)$ & 0.739 \\
\hline PET uptake $\left(\mathrm{SUV}_{\max } \geq 2.5\right)(n=20)$ & $18 / 20(90)$ & $8(40)$ & $10(50)$ & 0.224 \\
\hline Granuloma on histology & $20(25)$ & $5(16)$ & $15(31)$ & 0.115 \\
\hline
\end{tabular}

Data are presented as number (\%) or median (interquartile range). ${ }^{\mathbb{I}}$ Long-axis diameter. PET, positron emission tomography; $\mathrm{SUV}_{\max }$, maximum standardized uptake. 


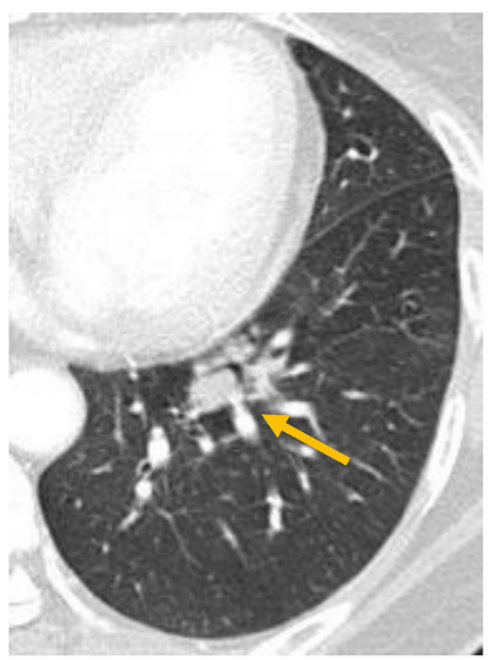

(A)

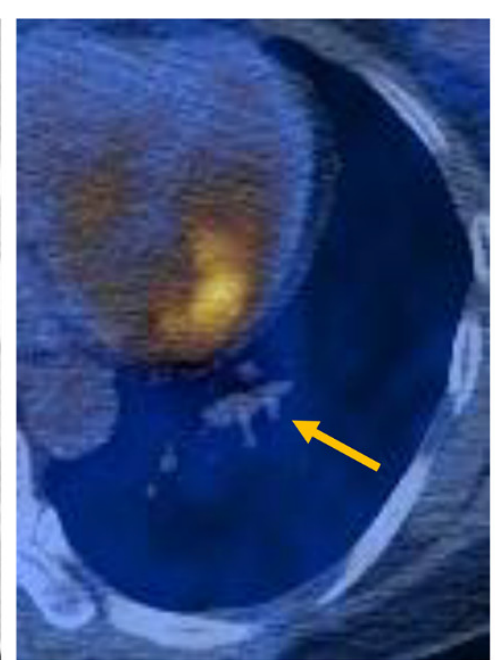

(B)

Figure 1. Cavitary Aspergillus nodule (arrowhead) from a 62-year-old woman. (A) Chest CT revealed a $23 \mathrm{~mm}$ round nodule in the left lower lobe. (B) PET/CT uptake showed a SUV max value of 2.0.

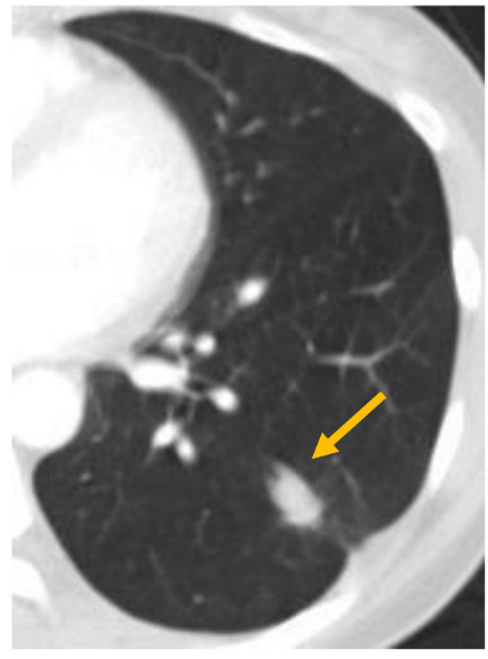

(A)

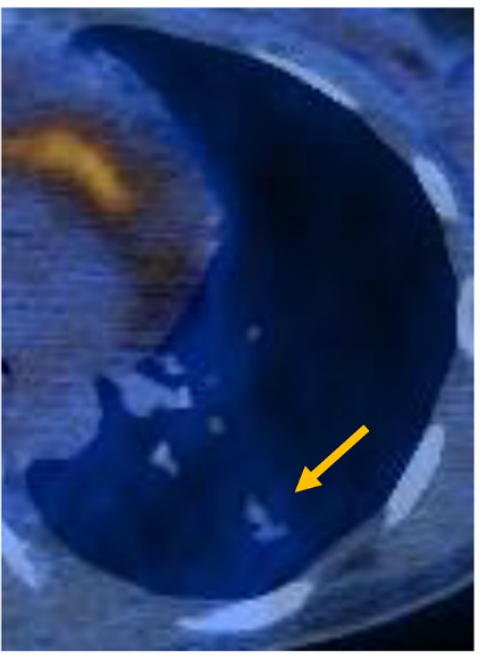

(B)

Figure 2. Non-cavitary Aspergillus nodule (arrowhead) from a 45-year-old woman. (A) Chest CT showed a $16 \mathrm{~mm}$ smooth oval nodule in the peripheral portion of the left lower lobe. (B) PET/CT uptake showed a SUV max value of 1.2.

\subsection{Treatment Modalities and Outcomes}

Treatment modalities and outcomes of patients with Aspergillus nodules are shown in Table 3. Of 73 patients who underwent surgical resection for Aspergillus nodule(s), most patients $(79 \%, 58 / 73)$ received no additional antifungal therapy, and approximately half of these patients $(52 \%, 30 / 58)$ had received sublobar resection. Fifteen patients $(21 \%, 15 / 73)$ who had surgical resection received subsequent additional antifungal therapy, and 12 of these 15 patients underwent lobectomy or sublobar resection. The nodules were incidentally confirmed in the remaining three of 15 patients. One patient underwent lung transplantation due to advanced interstitial pulmonary fibrosis related to Sjögren's disease. The other two patients underwent completion pneumonectomy, due to severe hemoptysis after prior lobectomy.

Five patients $(6 \%, 5 / 80)$ received antifungal therapy alone, and two patients $(3 \%, 2 / 80)$ received no additional treatment after confirmation of a diagnosis of Aspergillus nodule by PCNB, without surgical resection. Of the 73 patients who underwent surgical resection, surgery was performed via 
thoracotomy in 21 patients and video-assisted thoracoscopic surgery in 52 patients. Representative histological findings of Aspergillus nodules are shown in Figure 3.

Table 3. Treatment modalities and outcomes of patients with Aspergillus nodules.

\begin{tabular}{|c|c|c|c|c|}
\hline Characteristics & $\begin{array}{l}\text { Total } \\
n=80)\end{array}$ & $\begin{array}{l}\text { Non-Cavitary Nodules } \\
(n=31)\end{array}$ & $\begin{array}{l}\text { Cavitary Nodules } \\
(n=49)\end{array}$ & $p$-Value \\
\hline Modality for diagnosis & & & & 0.012 \\
\hline PCNB & $7(9)$ & $6(19)$ & $1(2)$ & \\
\hline Surgical resection & $73(91)$ & $25(81)$ & $48(98)$ & \\
\hline Treatment modality ${ }^{\mathbb{I}}$ & & & & 0.042 \\
\hline Surgery only & $58(73)$ & $19(61)$ & $39(80)$ & \\
\hline Lobectomy & $28 / 58(48)$ & $6 / 19(32)$ & $22 / 39(56)$ & \\
\hline Sublobar resection & $30 / 58(52)$ & $13 / 19(68)$ & $17 / 39(44)$ & \\
\hline Surgery + antifungal agent & $15(19)$ & $6(19)$ & $9(18)$ & \\
\hline Pneumonectomy & $2 / 15(13)$ & 0 & $2 / 9(22)$ & \\
\hline Lobectomy & $6 / 15(40)$ & $2 / 6(23)$ & $4 / 9(44)$ & \\
\hline Sublobar resection & $6 / 15(40)$ & $4 / 6(67)$ & $2 / 9(22)$ & \\
\hline Lung transplantation & $1 / 15(7)$ & 0 & $1 / 9(11)$ & \\
\hline Antifungal agent only§ & $5(6)$ & $4(13)$ & $1(2)$ & \\
\hline No treatment ${ }^{\S}$ & $2(3)$ & $2(6)$ & 0 & \\
\hline Antifungal agent $(n=20)$ & & & & 1.000 \\
\hline Itraconazole & $19 / 20(95)$ & $10(100)$ & $9(90)$ & \\
\hline Voriconazole & $1 / 20(5)$ & $0(0)$ & $1(10)$ & \\
\hline Postoperative complications & $3 / 73(4)$ & $1 / 25(4)$ & $2 / 48(4)$ & 1.000 \\
\hline Pneumothorax & $1 / 3(33)$ & $1(100)$ & 0 & \\
\hline Hemoptysis & $1 / 3(33)$ & 0 & $1 / 2(50)$ & \\
\hline Acute lung injury & $1 / 3(33)$ & 0 & $1 / 2(50)$ & \\
\hline Duration of antifungal agent & $5.3(2.3-8.5)$ & $4.8(1.9-8.0)$ & $5.3(2.6-9.0)$ & 0.586 \\
\hline Follow-up, months & $36.8(16.0-70.5)$ & $43.3(14.0-70.9)$ & $35.6(20.4-69.9)$ & 0.886 \\
\hline
\end{tabular}

Data are presented as number (\%) or median (interquartile range). II Surgery was performed via thoracotomy in 21 patients and video-assisted thoracoscopic surgery in 52 patients. One patient was diagnosed with interstitial lung disease related to Sjögren's syndrome and underwent bilateral lung transplantation due to interstitial lung disease; the presence of Aspergillus nodules was determined during lung transplantation. $\S$ Antifungal agent alone $(n=5)$ and no treatment $(n=2)$ groups were diagnosed via PCNB, not surgical resection. PCNB, percutaneous transthoracic cutting needle biopsy.

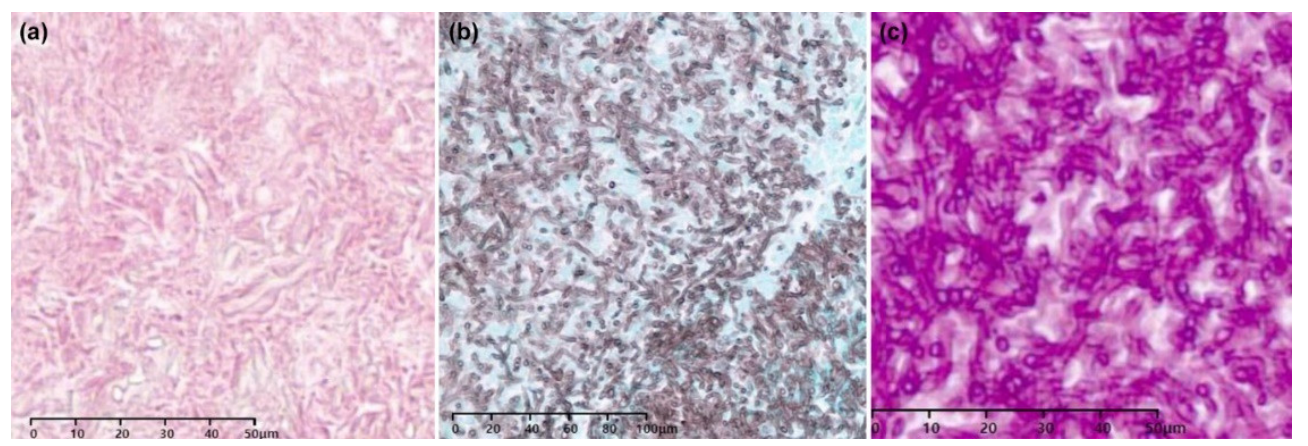

Figure 3. Surgical specimen of a non-cavitary Aspergillus nodule featuring dichotomous branching and hyphae with frequent septation. (a) Hyphae were observed as translucent material on hematoxylin and eosin staining. (b) Grocott's methenamine silver staining. (c) Periodic Acid-Schiff staining.

For the 20 patients who received antifungal therapy, the median duration of antifungal treatment was 5.3 months (interquartile range, 2.3-8.5 months). Most patients $(95 \%, 19 / 20)$ received itraconazole, while only one patient $(5 \%, 1 / 20)$ received voriconazole. Three patients experienced postoperative pulmonary complications: pneumothorax, hemoptysis, and acute lung injury ( $n=1$ each). There were no significant differences in postoperative complications between patients with non-cavitary nodules and patients with cavitary nodules.

There were no recurrences of Aspergillus infection during the median follow-up period of 36.8 months (interquartile range, 16.0-70.5 months). All seven of the patients who did not have resection had follow-up chest CT scans-two patients without treatment showed no change in the nodule after a one-year follow-up and the other five patients who had been treated with antifungals showed a decrease in nodule size in follow-up scans after treatment. Among the 10 patients who had 
positive results on Aspergillus precipitin IgG antibody tests before surgical resection, four patients had serial follow-up tests. Two patients had decreased titers after the surgical removal of their Aspergillus nodule(s), but showed elevated titers later on during follow-up, although there was no evidence of Aspergillus infection. The other two patients showed elevated antibody titers even after the removal of their Aspergillus nodules.

\section{Discussion}

Aspergillus nodules represent an uncommon subtype of CPA and are a clinical problem because they often mimic other diseases, such as malignancy or inflammatory nodules $[1,9,10]$. In this study, we comprehensively reviewed patients with pathologically confirmed Aspergillus nodules. Most of our patients were middle-aged with normal body mass index and had symptoms such as hemoptysis (68\%). Inflammatory markers showed nonspecific findings; however, the WBC count tended to be slightly higher in patients with cavitary Aspergillus nodules. The median diameter of the nodules was $<3 \mathrm{~cm}$, and most nodules had smooth margins $(80 \%)$ without calcification $(61 \%)$ on CT, although more than half $(52 \%)$ of the nodules were not visible on radiographs. There were no significant differences in clinical-radiological features or prognosis between patients with cavitary nodules and those with non-cavitary nodules. Attempts have been made to establish detailed features and treatment outcomes of Aspergillus nodules, but previous publications have largely consisted of case series or small observational studies $[9,10,12]$. Although a recent study performed in the U.K. involved 33 immunocompetent patients with Aspergillus nodules [8], only limited data are available regarding the characteristics of Aspergillus nodules. Therefore, our data provide insight in this context.

A notable finding of the present study was that patients with Aspergillus nodules had favorable outcomes, regardless of the presence or absence of internal cavitation. Most patients could be treated completely by surgical resection without perioperative antifungal therapy, and two of our patients received no treatment after Aspergillus nodules were diagnosed using a nonsurgical approach. Furthermore, no patients exhibited recurrent pulmonary Aspergillus infection during the median follow-up period of 36.8 months. These findings were consistent with the results of previous studies, in terms of favorable outcomes for patients with Aspergillus nodules [18]. Because Aspergillus nodules in the present study generally manifested as localized, solitary nodules, our data suggest that complete surgical resection of Aspergillus nodules may be curative in the absence of other remnant lesions presented radiographically. In addition, although small in number, our data may suggest that conservative management with close observation alone could be a treatment strategy worth considering, especially in patients for whom surgery is a high-risk approach.

With regard to antifungal therapy for Aspergillus nodules, limited data are available concerning optimal treatment duration or on the benefits of additional antifungal therapy after surgical resection $[2,19]$. Current guidelines indicate that when the Aspergillus nodule is single and completely resected, antifungal therapy is not required for non-immunocompromised patients [1]. However, a subset of patients with Aspergillus nodules may have chronic inflammatory pulmonary or extra-pulmonary disease, which requires treatment with immunomodulatory drugs. As no guidance has been established regarding a beneficial role for antifungal therapy, further studies are needed to determine treatment strategies with regard to the use of antifungal agents for the management of Aspergillus nodules. We cautiously suggest that the use of antifungal therapy could be individualized based on preoperative clinical symptoms or postoperative outcome.

In our study, postoperative complications occurred in three of the 73 patients who underwent surgery. One patient exhibited mild pneumothorax after hospital discharge and two patients had severe hemoptysis and acute lung injury. The patient with pneumothorax recovered quickly after the insertion of a chest tube, but the other two patients underwent segmentectomy/lobectomy and received mechanical ventilation in the intensive care unit. Although the postoperative complications for these two patients were eventually fatal, there were no mortalities during the study period and the incidence of therapy-related complications was lower than with other benign lung diseases [20-22]. Various 
complications associated with aspergilloma resection are known to occur, including persistent air leakage, empyema, pneumonia, bronchopleural fistula, respiratory failure, massive hemorrhage, and death [23]; thus, patients should be carefully selected before undergoing surgery. However, there is a lack of data regarding the outcomes of surgical treatment of Aspergillus nodules. Therefore, our data may provide more precise information regarding this topic.

In the current guidelines, the detection of serum Aspergillus precipitin IgG antibody is a key diagnostic criterion for CPA [1,24]. Unfortunately, it is unclear whether the presence of Aspergillus IgG antibody could also be a supportive finding in the diagnosis of Aspergillus nodules. In the present study, only a small number of patients had been tested for serum Aspergillus precipitin IgG antibody; however, fewer than half $(10 / 24,42 \%)$ of these patients had positive results. These observations suggest that the Aspergillus IgG antibody test may be unsuitable for the diagnosis of Aspergillus nodules, due to its low sensitivity. Similarly, Muldoon et al. reported that Aspergillus precipitin was positive in only four of 32 patients with Aspergillus nodules [8]. Thus, further studies are needed to clearly establish the role of the test in the diagnosis of Aspergillus nodules.

In our study, Aspergillus nodules showed no distinctive features on chest CT that could enable them to be distinguished from malignant or other inflammatory nodules. In addition, $90 \%(18 / 20)$ of the Aspergillus nodules for which data were available showed high SUV $\mathrm{max}_{\text {ux }}$ uptake on FDG-PET/CT. As malignant nodules or granulomatous nodules, due to tuberculosis or nontuberculous mycobacteria, can show positive signals on PET [25], our data suggested that radiological imaging alone is unlikely to be useful for the differentiation of Aspergillus nodules from other etiologies [26]. Thorough investigations, including preoperative histological confirmation, should be considered before undertaking surgical resection to avoid unnecessary intervention or the misdiagnosis of benign conditions $[9,26]$.

This study had several potential limitations. First, it was a single-center, retrospective study. However, this is the largest study in patients with Aspergillus nodules reported thus far, despite the low prevalence of CPA. Second, the serum Aspergillus precipitin IgG antibody test was performed in less than half of the study population. Although the low sensitivity may have been overestimated, due to the small number of patients, the low sensitivity of the serum Aspergillus precipitin IgG antibody test indicates the need for novel biomarkers for the diagnosis of Aspergillus nodules. Third, the follow-up period may have been insufficient to evaluate long-term prognosis. Fourth, because approximately half of our patients had an underlying respiratory disease, some of the presented symptoms in our study patients may not be caused by Aspergillus nodules, but by underlying diseases. Lastly, as our study population was based on patients who could undergo invasive procedures, there may be a selection bias.

In conclusion, surgery could be a treatment strategy worth considering for most Aspergillus nodules. However, given that our study population was heterogeneous, further well-designed studies are need.

Author Contributions: Study conception and design: N.K., J.P., and B.W.J.; data acquisition and analysis: N.K., J.P., and B.W.J.; data interpretation and manuscript writing: N.K., J.P., and B.W.J.; critical revision and final approval of the manuscript: N.K., J.P., and B.W.J. All authors have read and agreed to the published version of the manuscript.

Funding: This research received no external funding.

Acknowledgments: The authors would like to express our deepest gratitude and respect to Won-Jung Koh for his invaluable guidance, unfailing encouragement, and critical insight. As one of the best physicians and researchers, he encouraged us to develop this work. Won-Jung Koh passed away in August 2019. We dedicate this work to his memory.

Conflicts of Interest: The authors declare no conflict of interest. 


\section{References}

1. Denning, D.W.; Cadranel, J.; Beigelman-Aubry, C.; Ader, F.; Chakrabarti, A.; Blot, S.; Ullmann, A.J.; Dimopoulos, G.; Lange, C. Chronic pulmonary aspergillosis: Rationale and clinical guidelines for diagnosis and management. Eur. Respir. J. 2016, 47, 45-68. [CrossRef]

2. Patterson, T.F.; Thompson, G.R., 3rd; Denning, D.W.; Fishman, J.A.; Hadley, S.; Herbrecht, R.; Kontoyiannis, D.P.; Marr, K.A.; Morrison, V.A.; Nguyen, M.H.; et al. Practice Guidelines for the Diagnosis and Management of Aspergillosis: 2016 Update by the Infectious Diseases Society of America. Clin. Infect. Dis. 2016, 63, e1-e60. [CrossRef]

3. Page, I.D.; Byanyima, R.; Hosmane, S.; Onyachi, N.; Opira, C.; Richardson, M.; Sawyer, R.; Sharman, A.; Denning, D.W. Chronic pulmonary aspergillosis commonly complicates treated pulmonary tuberculosis with residual cavitation. Eur. Respir. J. 2019, 53, 1801184. [CrossRef] [PubMed]

4. Smith, N.L.; Denning, D.W. Underlying conditions in chronic pulmonary aspergillosis including simple aspergilloma. Eur. Respir. J. 2011, 37, 865-872. [CrossRef]

5. Bongomin, F.; Harris, C.; Foden, P.; Kosmidis, C.; Denning, D.W. Innate and Adaptive Immune Defects in Chronic Pulmonary Aspergillosis. J. Fungi 2017, 3, 26. [CrossRef] [PubMed]

6. Brown, G.D.; Denning, D.W.; Gow, N.A.; Levitz, S.M.; Netea, M.G.; White, T.C. Hidden killers: Human fungal infections. Sci. Transl. Med. 2012, 4, 165rv113. [CrossRef] [PubMed]

7. Denning, D.W.; Pleuvry, A.; Cole, D.C. Global burden of chronic pulmonary aspergillosis as a sequel to pulmonary tuberculosis. Bull. World Health Organ. 2011, 89, 864-872. [CrossRef]

8. Muldoon, E.G.; Sharman, A.; Page, I.; Bishop, P.; Denning, D.W. Aspergillus nodules; another presentation of Chronic Pulmonary Aspergillosis. BMC Pulm. Med. 2016, 16, 123. [CrossRef]

9. Yasuda, M.; Nagashima, A.; Haro, A.; Saitoh, G. Aspergilloma mimicking a lung cancer. Int. J. Surg. Case Rep. 2013, 4, 690-692. [CrossRef]

10. Ahn, B.C.; Lee, S.W.; Lee, J.; Kim, C. Pulmonary aspergilloma mimicking metastasis from papillary thyroid cancer. Thyroid 2011, 21, 555-558. [CrossRef]

11. Baxter, C.G.; Bishop, P.; Low, S.E.; Baiden-Amissah, K.; Denning, D.W. Pulmonary aspergillosis: An alternative diagnosis to lung cancer after positive [18F]FDG positron emission tomography. Thorax 2011, 66, 638-640. [CrossRef] [PubMed]

12. Kang, E.Y.; Kim, D.H.; Woo, O.H.; Choi, J.A.; Oh, Y.W.; Kim, C.H. Pulmonary aspergillosis in immunocompetent hosts without underlying lesions of the lung: Radiologic and pathologic findings. AJR Am. J. Roentgenol. 2002, 178, 1395-1399. [CrossRef] [PubMed]

13. Baxter, C.G.; Denning, D.W.; Jones, A.M.; Todd, A.; Moore, C.B.; Richardson, M.D. Performance of two Aspergillus IgG EIA assays compared with the precipitin test in chronic and allergic aspergillosis. Clin. Microbiol. Infect. 2013, 19, E197-E204. [CrossRef]

14. Dumollard, C.; Bailly, S.; Perriot, S.; Brenier-Pinchart, M.P.; Saint-Raymond, C.; Camara, B.; Gangneux, J.P.; Persat, F.; Valot, S.; Grenouillet, F.; et al. Prospective Evaluation of a New Aspergillus IgG Enzyme Immunoassay Kit for Diagnosis of Chronic and Allergic Pulmonary Aspergillosis. J. Clin. Microbiol. 2016, 54, 1236-1242. [CrossRef] [PubMed]

15. Page, I.D.; Richardson, M.D.; Denning, D.W. Comparison of six Aspergillus-specific IgG assays for the diagnosis of chronic pulmonary aspergillosis (CPA). J. Infect. 2016, 72, 240-249. [CrossRef]

16. Hansell, D.M.; Bankier, A.A.; MacMahon, H.; McLoud, T.C.; Muller, N.L.; Remy, J. Fleischner Society: Glossary of terms for thoracic imaging. Radiology 2008, 246, 697-722. [CrossRef]

17. Mennink-Kersten, M.A.; Donnelly, J.P.; Verweij, P.E. Detection of circulating galactomannan for the diagnosis and management of invasive aspergillosis. Lancet Infect. Dis. 2004, 4, 349-357. [CrossRef]

18. Lee, S.H.; Lee, B.J.; Jung, D.Y.; Kim, J.H.; Sohn, D.S.; Shin, J.W.; Kim, J.Y.; Park, I.W.; Choi, B.W. Clinical manifestations and treatment outcomes of pulmonary aspergilloma. Korean J. Intern. Med. 2004, 19, 38-42. [CrossRef] [PubMed]

19. Zheng, S.; Li, X.; Hu, B.; Li, H. Is adjuvant antifungal therapy after video-assisted thoracic surgery for pulmonary aspergilloma necessary? J. Thorac. Dis. 2018, 10, 6060-6065. [CrossRef] [PubMed]

20. Kang, M.W.; Kim, H.K.; Choi, Y.S.; Kim, K.; Shim, Y.M.; Koh, W.J.; Kim, J. Surgical treatment for multidrug-resistant and extensive drug-resistant tuberculosis. Ann. Thorac. Surg. 2010, 89, 1597-1602. [CrossRef] [PubMed] 
21. Kwon, Y.S.; Kim, Y.H.; Suh, G.Y.; Chung, M.P.; Kim, H.; Kwon, O.J.; Choi, Y.S.; Kim, K.; Kim, J.; Shim, Y.M.; et al. Treatment outcomes for HIV-uninfected patients with multidrug-resistant and extensively drug-resistant tuberculosis. Clin. Infect. Dis. 2008, 47, 496-502. [CrossRef] [PubMed]

22. Mitchell, J.D.; Bishop, A.; Cafaro, A.; Weyant, M.J.; Pomerantz, M. Anatomic lung resection for nontuberculous mycobacterial disease. Ann. Thorac. Surg. 2008, 85, 1887-1892. [CrossRef] [PubMed]

23. Brik, A.; Salem, A.M.; Kamal, A.R.; Abdel-Sadek, M.; Essa, M.; El Sharawy, M.; Deebes, A.; Bary, K.A. Surgical outcome of pulmonary aspergilloma. Eur. J. Cardiothorac. Surg. 2008, 34, 882-885. [CrossRef] [PubMed]

24. Ma, X.; Wang, K.; Zhao, X.; Liu, Y.; Li, Y.; Yu, X.; Li, C.; Denning, D.W.; Xie, L. Prospective study of the serum Aspergillus-specific IgG, IgA and IgM assays for chronic pulmonary aspergillosis diagnosis. BMC Infect. Dis. 2019, 19, 694. [CrossRef]

25. Deppen, S.; Putnam, J.B., Jr.; Andrade, G.; Speroff, T.; Nesbitt, J.C.; Lambright, E.S.; Massion, P.P.; Walker, R.; Grogan, E.L. Accuracy of FDG-PET to diagnose lung cancer in a region of endemic granulomatous disease. Ann. Thorac. Surg. 2011, 92, 428-432, discussion 433. [CrossRef]

26. Ito, Y.; Tanigawa, M.; Takemura, T.; Iwamoto, K.; Nigi, A.; Sasano, H.; Itani, H.; Kondo, S.; Tokui, T.; Nakamura, S.; et al. Chronic Nodular Pulmonary Aspergillosis in a Patient with Rheumatoid Arthritis. Intern. Med. 2019, 58, 979-984. [CrossRef]

(C) 2020 by the authors. Licensee MDPI, Basel, Switzerland. This article is an open access article distributed under the terms and conditions of the Creative Commons Attribution (CC BY) license (http://creativecommons.org/licenses/by/4.0/). 